\title{
A new variable stiffness colonoscope makes colonoscopy easier: a randomised controlled trial
}

\author{
J C Brooker, B P Saunders, S G Shah, C B Williams
}

\begin{abstract}
Background-Colonoscopy remains technically difficult in $10-20 \%$ of procedures due to variable colonic anatomy and fixation. The ability to vary endoscope shaft flexibility may help insertion to the caecum.

Methods-Consecutive patients attending for day case colonoscopy were randomised to examination with either the conventional Olympus CF200HL (200HL) or a new variable stiffness (VS) colonoscope. Intubation time, use of stiffening function, and patient pain scores were compared.

Results-Of 100 cases, 43 were performed with the $200 \mathrm{HL}$ and 57 with the VS. Four incomplete examinations occurred with the 200HL (two sigmoid fixations, two benign strictures) and two with the VS (one obstructing cancer, one fixed sigmoid). Changing to the paediatric scope was successful in all but one patient from each group (obstructive lesions). Stiff mode was applied 23 times in 18 patients and was effective in 15 of these. Intubation time was quicker with the VS (median 6 minutes 32 seconds) than with the $200 \mathrm{HL}$ (median 10 minutes 35 seconds) $(p=0.0005)$. Pain scores were less with the VS (median 7) than with the $200 \mathrm{HL}$ (median 24) $(p=0.0081)$.

Conclusions-The variable stiffness colonoscope combines paediatric shaft characteristics with the ability to stiffen when needed. This instrument significantly reduces intubation time and patient discomfort. Further comparisons should be made with the newest colonoscopes which are less stiff.

(Gut 2000;46:801-805)
\end{abstract}

Keywords: colonoscopy; colonoscopes; technology; pain

It has been shown that difficult colonoscopies are often the result of recurrent loop formation in a long and mobile colon or sigmoid colon fixation secondary to diverticulosis or previous surgery. ${ }^{1}$ Until now the type of colonoscope best suited to each of these situations has had mutually exclusive characteristics. A stiffer colonoscope shaft reduces recurrent looping but may make passage through an angulated sigmoid colon more difficult and cause more stretch and pain when loops do occur. The more flexible paediatric instrument is better for negotiating a fixed or narrowed sigmoid colon but tends to allow recurrent loop formation ${ }^{2}$; it may also improve patient tolerance, ${ }^{2}$ prob- ably by reducing the amount of stretch caused by looping. ${ }^{4}$

Several methods have been proposed for varying colonoscope shaft stiffness. An overtube $^{5}$ is often successful in controlling sigmoid colon looping but cannot prevent proximal loops, is cumbersome to use, and may cause complications. ${ }^{6}$ Success has been reported with stiffening wires inserted through the biopsy channel ${ }^{7-9}$ but their use prolongs the examination, disables the suction/instrumentation channel, and can also damage the colonoscope. ${ }^{10} \mathrm{~A}$ method is also described for exchanging between paediatric and conventional instruments over a guidewire, after

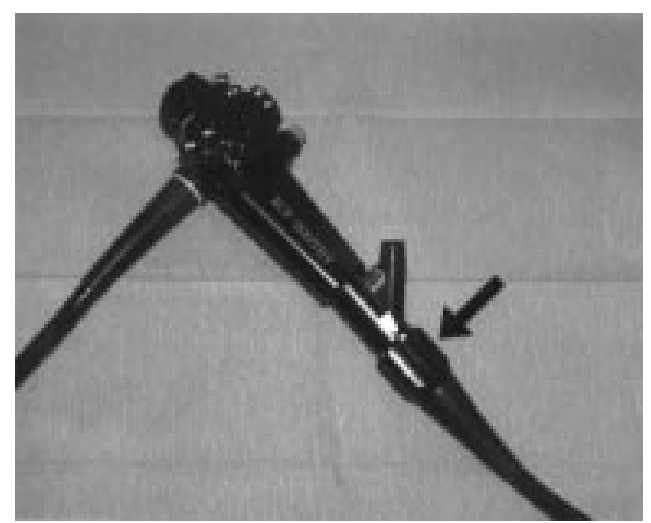

Figure 1 The control head of the variable stiffness colonoscope (Olympus XCF-SH23OL). The dial for controlling the stiffness is arrowed. Specifications: distal end outer diameter $13.6 \mathrm{~mm}$, flexible tube outer diameter $12.9 \mathrm{~mm}$, instrument channel $3.2 \mathrm{~mm}$, and working length $168 \mathrm{~cm}$.

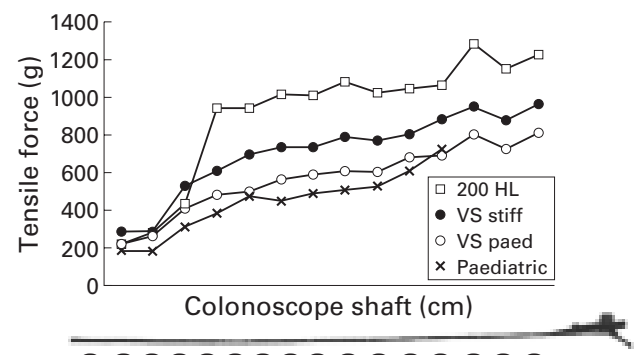

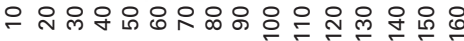

Figure 2 The stiffness of three colonoscope shafts: the conventional colonoscope (200HL), the variable stiffness colonoscope in paediatric mode (VS paed) and at maximum stiffness (VS stiff), and the paediatric colonoscope. Stiffness was measured at $10 \mathrm{~cm}$ intervals along the shaft from the $20 \mathrm{~cm}$ mark using a tension gauge to depress each point by $5 \mathrm{~cm}$ when suspended across a 20 $\mathrm{cm}$ gap. Each value is the mean of three readings.

Abbreviations used in this paper: VS colonoscope, variable stiffness colonoscope; $\mathrm{MEI}$, magnetic endoscope imaging. 
Table 1 Patient characteristics in the groups randomised to be examined with the variable stiffness (VS) and conventional (200HL) colonoscopes

\begin{tabular}{lll}
\hline & $V S(n=57)$ & $200 H L(n=43)$ \\
\hline Age (median (range)) $\neq$ & $58(26-82)$ & $55(24-86)$ \\
Female $^{\star}$ & 33 & 29 \\
Aale $^{\star}$ & 24 & 14 \\
Hyxiety (median (range)) $\dagger$ & $31(0-95)$ & $29(0-100)$ \\
Diverticular disease & 1 & 3 \\
\hline
\end{tabular}

$\neq \mathrm{p}=0.65$ (Mann-Whitney); ${ }^{\star} \mathrm{p}=0.4$ (Fisher's exact); $\uparrow \mathrm{p}=0.95$ (Mann-Whitney); †tp $=0.3116$ (Fisher's exact); ${ }^{\star \star} \mathrm{p}=0.1985$ (Fisher's exact).

Table 2 Indications for colonoscopy in the groups examined with the variable stiffness (VS) and conventional (200HL) colonoscopes

\begin{tabular}{llll}
\hline & $\begin{array}{l}\text { VS } \\
(n=57)\end{array}$ & $\begin{array}{l}200 H L \\
(n=43)\end{array}$ & $\begin{array}{l}p \text { (Fisher's } \\
\text { exact test) }\end{array}$ \\
\hline IBD & 22 & 10 & 0.1311 \\
Bowel symptoms & 20 & 20 & 0.3041 \\
Cancer/polyp screening & 15 & 13 & 0.8223
\end{tabular}

IBD, assessment of extent of inflammatory bowel disease, extensive ulcerative colitis surveillance, or differential diagnosis. Bowel symptoms, change in bowel habit, rectal bleeding, abdominal pain, anaemia, or abnormal barium.

Cancer/polyp screening, family history of colon cancer or past history of polyps or cancer.

passage through the sigmoid, ${ }^{3}$ but this is time consuming and requires fluoroscopy.

Our hypothesis was that a new colonoscope with in-built variable shaft stiffness should improve intubation time and reduce patient discomfort during insertion (by virtue of increased flexibility in the paediatric setting), without adversely affecting completion rates (because of the ability to avoid reformation of loops after straightening by increasing the shaft stiffness).

\section{Materials and methods}

VARIABLE STIFFNESS (VS) COLONOSCOPE

The prototype colonoscope (Olympus XCFSH230L) has a graduated dial situated just below the control head of the colonoscope (fig 1) which can be turned manually to vary shaft stiffness from minimum to maximum. In all other respects it is a normal video colonoscope (shaft diameter $12.9 \mathrm{~mm}$, working length 168 $\mathrm{cm}$, instrument channel $3.2 \mathrm{~mm}$ ). The mechanism of adjustable shaft stiffness is similar to that used in commercially available "through the channel" stiffening wires. These have a central cable with a surrounding metal helical coil. Tension applied to the cable compresses and stiffens the helix and colonoscope, whereas loosening has the opposite effect resulting in paediatric characteristics. The stiffening mechanism terminates $30 \mathrm{~cm}$ from the instrument

Table 3 Incomplete examinations. Cases in which caecal intubation with the designated colonoscope was unsuccessful, listed in order of occurrence. The colonoscope used, reason for failure, and result of converting to the paediatric colonoscope are also given

\begin{tabular}{llll}
\hline Case & Colonoscope & Reason for failure & $\begin{array}{l}\text { Completed with } \\
\text { paediatric } \\
\text { colonoscope }\end{array}$ \\
\hline 31 & VS & Obstructing sigmoid cancer & No \\
57 & $200 \mathrm{HL}$ & Fixed sigmoid angulation & Yes \\
70 & VS & Fixed sigmoid (diverticular disease) & Yes \\
81 & $200 \mathrm{HL}$ & NSAID induced diaphragm (stricture) transverse colon & No \\
89 & $200 \mathrm{HL}$ & Fixed sigmoid (diverticular disease) & Yes \\
95 & $200 \mathrm{HL}$ & Rectosigmoid stricture (Crohn's) & Yes \\
\hline
\end{tabular}

${ }^{\star} \mathrm{p}=0.3979$ (Fisher's exact) tip so that the distal portion remains relatively floppy. For the purposes of this study minimum stiffness was used as the default setting. When greater shaft stiffness was required the maximum setting was used.

\section{CONTROL COLONOSCOPE}

The control instrument used in this study was the standard Olympus CF200HL (200HL): shaft diameter $13.3 \mathrm{~mm}$, instrument channel $3.2 \mathrm{~mm}$, working length $165 \mathrm{~cm}$. This colonoscope was selected because it was the most widely used in UK endoscopy departments in early 1998 (personal communications, Olympus Keymed, UK) when the study began.

The Olympus PCF 230 paediatric colonoscope (shaft diameter $11.3 \mathrm{~mm}$, instrument channel $3.2 \mathrm{~mm}$, working length $130 \mathrm{~cm}$ ) was used to attempt completion in cases that had failed due to fixation or stricture.

The shaft stiffness of the variable stiffness, 200HL, and PCF colonoscopes was measured for comparison. A tension gauge was used at $10 \mathrm{~cm}$ intervals to determine the force required (mean of three readings) to depress the shaft by $5 \mathrm{~cm}$ when suspended between two points $20 \mathrm{~cm}$ apart (fig 2).

\section{PATIENTS}

Consecutive patients attending for routine day case colonoscopy were randomised (random number generator) to be examined with either the $200 \mathrm{HL}$ or VS colonoscope. Patients and admission/recovery nurses were blinded to the type of colonoscope used. Patients also scheduled for gastroscopy or those with previous colonic resection were excluded. All patients were given routine bowel preparation: 24 hour dietary restriction, magnesium citrate $(29.5 \mathrm{~g}$ sachets $\times 2$ ) and senna granules (13 g weight). Pre-procedure anxiety was assessed with a 100 $\mathrm{mm}$ visual analogue scale $(0=$ not worried, $100=$ extremely anxious) completed by the patient after explanation by the admitting nurse. Patients who requested sedation were given standard initial intravenous medication with midazolam $1.25 \mathrm{mg}$, pethidine $25 \mathrm{mg}$, and hyoscine $20 \mathrm{mg}$. Additional doses were given as required. Each procedure was performed by one of two experienced colonoscopists (CBW and BPS). All examinations were commenced in the left lateral position. Caecal intubation time was recorded as the interval between initial insertion through the anal canal and intubation of the caecal pole, assessed by recognition of the appendix orifice and ileocaecal valve. Cases in which caecal intubation failed with the allocated instrument were counted as failed examinations. Data from these cases were excluded from the analysis of intubation time and pain. The paediatric colonoscope was used to attempt completion in those cases that failed with either of the randomised instruments.

The maximum stiffness setting of the VS scope was used only when shaft looping occurred at the default (minimum stiffness) setting and did not respond to abdominal hand pressure or change in position. Maximum stiffness was only applied after fully straightening 


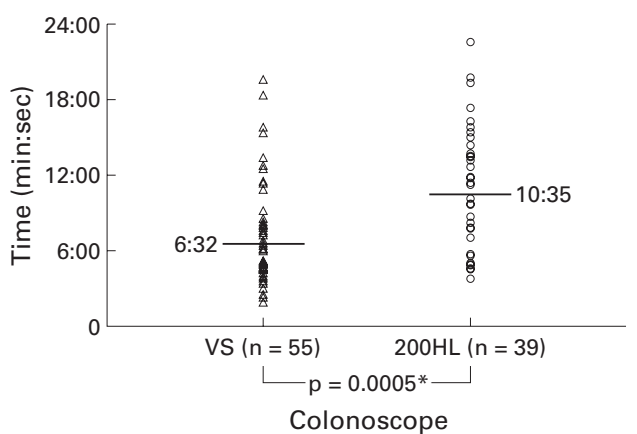

Figure 3 Caecal intubation times and median values for examinations completed with the variable stiffness (VS) and conventional (200HL) colonoscopes. ${ }^{\star}$ Mann Whitney $U$ test.

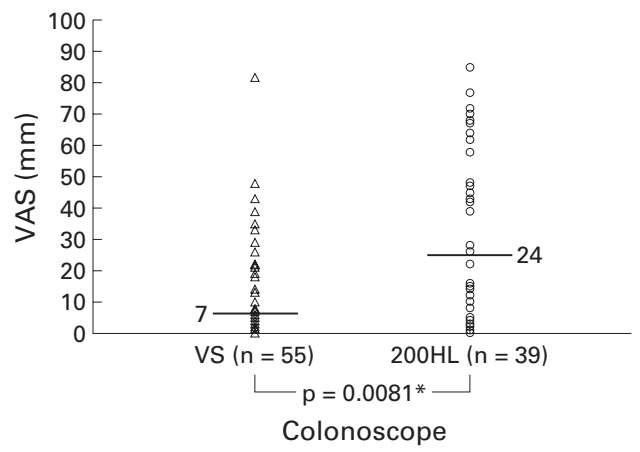

Figure 4 Pain scores assessed using a $100 \mathrm{~mm}$ visual analogue scale for examinations completed with the variable stiffness (VS) and conventional (200HL) colonoscopes. *Mann Whitney U test.

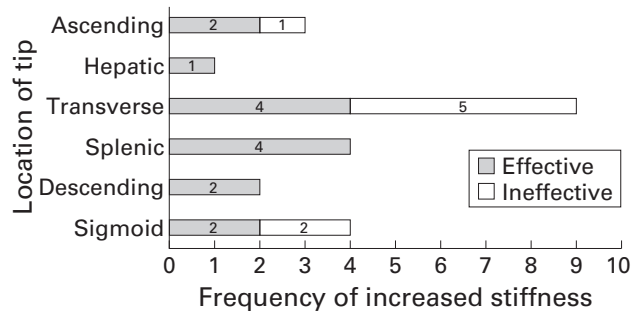

Figure 5 Efficacy of stiffening the colonoscope shaft in overcoming recurrent looping according to the estimated location of the tip.

Table 4 Male and female pain scores. Patient pain scores (in examinations that were completed with the assigned instrument) for males and females for the variable stiffness (VS) and control (200HL) colonoscopes

\begin{tabular}{llcc}
\hline & Male (range) $[n]$ & Female (range) $[n]$ & $p$ Value \\
\hline 200HL & $8(0-70)[13]$ & $39(1-85)[27]$ & 0.0122 \\
VS & $5.5(0-48)[23]$ & $9(2-82)[31]$ & 0.3832 \\
p Value* & 0.9119 & 0.0022 &
\end{tabular}

^Mann-Whitney U test.

Table 5 Male and female caecal intubation times. Caecal intubation times (in examinations that were completed with the assigned instrument) for males with females for the variable stiffness (VS) and control (200HL) colonoscopes

\begin{tabular}{llll}
\hline & Male (range) $[n]$ & Female (range) $[n]$ & $p$ Value $^{*}$ \\
\hline 200HL & $07: 40(3: 50-13: 40)[13]$ & $11: 45(4: 33-22: 33)[27]$ & 0.0136 \\
VS & $4: 48(1: 55-19: 40)[23]$ & $7: 26(3: 07-18: 14)[31]$ & 0.0038 \\
p Value & 0.0395 & 0.0038 & \\
\hline
\end{tabular}

Times are in minutes:seconds.

*Mann-Whitney U test. the instrument shaft. Whenever shaft stiffening was used, colonoscope tip location was estimated as well as the efficacy of the manoeuvre. A successful result was considered to be immediate advancement of the colonoscope tip. After the manoeuvre the VS colonoscope was returned to minimum stiffness. A $100 \mathrm{~mm}$ visual analogue scale for pain ( $0=$ painless, $100=$ extremely painful) during colonoscopy was completed by the patients immediately prior to discharge, usually within 45 minutes of completing the examination.

\section{STATISTICAL ANALYSIS}

Differences in anxiety score, pain score, and intubation time were compared using the Mann-Whitney $U$ test. The characteristics of the two groups of patients were compared using Fisher's exact test. All $\mathrm{p}$ values were twotailed and $p<0.05$ was considered significant.

\section{Results}

One hundred consecutive eligible patients were randomised. There was no significant difference between the two patient groups in age, sex, anxiety, previous diverticular disease, or hysterectomy (table 1). There was also no difference in indications for colonoscopy (table 2) or amount of sedation used (VS group: median pethidine dose $25 \mathrm{mg}$ (range 0-75), median midazolam dose $1.5 \mathrm{mg}(0-3) ; 200 \mathrm{HL}$ group:

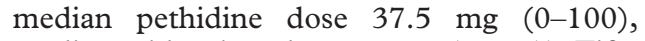
median midazolam dose $1.5 \mathrm{mg}(0-2.5)$ ). Fifty five cases were completed with the VS and $39 / 43$ with the $200 \mathrm{HL}$ colonoscopes. There were no complications in either group.

The six incomplete examinations are detailed in table 3. Four were the result of benign fixation of the sigmoid (two diverticular disease, one Crohn's disease, one no cause stated). All were completed after changing to the paediatric colonoscope. The other two failures, an obstructing sigmoid cancer and a tight NSAID induced transverse colon stricture, could not be passed even with the paediatric colonoscope.

Of the completed examinations, intubation time was significantly quicker $(p=0.0005)$ using the VS colonoscope (fig 3) (VS: median time 6 minutes 32 seconds (range 1:50-19:35); 200HL: median time 10 minutes 35 seconds (3:45-22:35)). Patient pain was significantly less $(p=0.0081)$ with the VS colonoscope (fig 4) (VS: median pain score 7 (range 0-82); 200HL: median pain score 24 (0-85)). During the examinations performed with the VS scope, shaft stiffness was increased because of uncontrollable looping 23 times in 18 patients and was considered to be effective on 15 of 23 occasions (fig 5). Analysis by patient sex is detailed in tables 4 and 5. Women recorded significantly more pain than men with the $200 \mathrm{HL}$ but there was no difference between the sexes when the VS colonoscope was used. Intubation times were quicker in men than women for both instruments, and quicker in women with the VS colonoscope than with the $200 \mathrm{HL}$. 


\section{Discussion}

A fixed sigmoid colon or recurrent looping are the commonest difficulties that arise during colonoscope insertion and may coexist in the same patient. ${ }^{1}$ The flexibility and narrow calibre of a paediatric colonoscope enables an easier, less traumatic passage through a narrowed or angulated colon. ${ }^{2}{ }^{4}$ However, the tendency of the paediatric instrument to form loops and its limited length $(130 \mathrm{~cm})$ compared with a standard colonoscope $(160 \mathrm{~cm})$ reduces the frequency of caecal intubation. ${ }^{3}$ In most cases the anatomy and characteristics of a patient's colon are not known before starting the procedure. It is only when difficulty occurs or the patient experiences excessive pain with a standard colonoscope that the need to consider changing to the paediatric instrument becomes apparent.

In the patient with a redundant or mobile colon, recurrent looping commonly occurs even with the stiffer conventional adult colonoscope. Devices for increasing shaft stiffness without the need for a change of instrument have included a wire inserted through the operating channel" ${ }^{7-9}$ and the "splinting" overtube $^{11}$ or its split version..$^{5}$ Although effective, both of these approaches have drawbacks. Stiffening wires obstruct the suction/biopsy channel thus impairing performance. They may also damage the colonoscope. ${ }^{10}$ An overtube is cumbersome, often requires fluoroscopy for effective use, may cause complications such as perforation, ${ }^{11}$ and even if successful in the sigmoid colon it does not prevent proximal looping.

In this study we have evaluated a new colonoscope which combines the shaft flexibility of a paediatric colonoscope with the working length of an adult colonoscope and a built in mechanism for stiffening the shaft when looping occurs. Thus the shaft characteristics of the VS colonoscope can be adapted to suit the fixed or looping colon without wasting time or compromising the procedure and with no risk of damaging the patient or instrument.

We have shown that the new VS colonoscope significantly reduces intubation time and patient discomfort compared with a standard adult instrument. The reduction in intubation time was probably the result of two main factors. Firstly, being more flexible, the prototype instrument traversed the sigmoid colon more easily. Secondly, when looping did not respond to hand pressure or position change, straightening the shaft and increasing the stiffness resulted in better progress on 15 of 23 occasions. Because the endoscopists could not be blinded, it is possible that they unintentionally tried harder with the new instrument. However, it is unlikely that this bias alone could have accounted for the magnitude of difference that we observed between the two scopes.

The reduction in pain score recorded by the patient was probably the result of less stretching of sigmoid colon loops by the floppy shaft of the VS colonoscope when in paediatric mode. The shorter procedure time may also have contributed. A much larger study is needed to determine whether reducing the stretching forces exerted on the sigmoid colon and attachments also lessen the risk of perforation during diagnostic colonoscopy with this instrument. Although we showed no difference in sedation requirements between the two groups, lessening the trauma of insertion through the sigmoid may reduce, and in some cases eliminate, the need for medication. This should encourage the increasingly popular practice of non-sedated colonoscopy ${ }^{12}$ with a consequent reduction of cardiorespiratory complications.

It is well documented ${ }^{11}$ that colonoscopy is more difficult, takes longer, and is less well tolerated in women than men. This is probably because of the longer female colon, ${ }^{11}{ }^{13}$ increased incidence of pelvic adhesions, and perhaps greater sensitivity to pain in the sigmoid colon. The results of analysis by patient sex are therefore of interest. We have shown that, with the new instrument, intubation times are reduced by an average of 3-4 minutes in both men and women, and that pain scores in females are reduced to levels experienced by men.

Caecal intubation was not achieved with the designated colonoscope in six cases, two with the VS and four with the $200 \mathrm{HL}$ colonoscope. Only one case in each group, caused by obstructing lesions, could not be completed after changing to a paediatric colonoscope. The numbers in this study are insufficient to show a significant difference in completion between the two instruments. However, it is logical that the floppier VS colonoscope should be able to negotiate tight angulations or fixed loops more easily. On one occasion in this study, a fixed sigmoid could only be successfully intubated after exchanging the VS for a narrower, floppier paediatric colonoscope. It might therefore be argued that a modified VS colonoscope, with a similar calibre to that of the paediatric instrument, if used routinely would obviate the need to change instruments for a very narrowed or fixed sigmoid colon. However, in pilot studies we have found that difficulty gripping the narrow shaft of such an instrument makes routine use less practical. Thus the more flexible, narrow calibred, paediatric colonoscope with its unique bending section characteristics is likely to remain an important back up for the occasional very narrowed or fixed sigmoid colon.

We have shown that the stiffening function of the VS scope was effective 15/23 times overall, with varying degrees of success at different locations. It was used most often at locations where looping most commonly occurs - that is, the sigmoid colon, splenic flexure, and transverse colon-where it was effective on $2 / 4,4 / 4$, and 4/9 occasions, respectively. The effectiveness of increasing shaft stiffness will probably improve with practice as endoscopists become adept at identifying the circumstances in which it will help insertion. We have previously shown ${ }^{14}$ that even experienced endoscopists are frequently inaccurate when estimating colonoscope location and loop configuration. This might confound the utility of the variable stiffness function in some cases. However, new 
developments in imaging technology, such as magnetic endoscope imaging (MEI $)^{15}$ which shows the colonoscope configuration in three dimensions, will enable the user to determine the precise nature and location of a loop. The addition of MEI may therefore further enhance the effect of adjusting shaft stiffness, allowing optimal straightening of the shaft before increased stiffness is applied. The availability, in the near future, of colonoscopes that integrate "variable stiffness" and built in MEI sensor coils, will allow these two features to be evaluated simultaneously.

For safety reasons the developers of the VS scope have limited the maximum stiffness available. Our own measurements have shown that even at the highest setting the stiffness is less than that of the CF200HL. In our experience even the relatively stiff conventional adult colonoscope is frequently not stiff enough to prevent looping. The design of the VS colonoscope will therefore probably need to be modified to increase the range of stiffness available.

As colonoscopic surveillance and population screening for colorectal cancer gain wider acceptance ${ }^{16}$ the number of colonoscopies performed on asymptomatic, healthy individuals will rise. This will create even more demand for quick, accurate, comfortable, and safe colonoscopy. We believe that this innovative instrument will be a valuable tool in achieving these aims and will probably become the colonoscope of first choice. Further studies will determine if the VS colonoscope confers the same or even more pronounced advantages when used by non-experts.

In conclusion, the new variable stiffness colonoscope improves the performance of colonoscopy in expert hands by reducing intubation time and patient discomfort. Modifications that may enhance the efficacy further include more floppiness in the paediatric setting and greater stiffness at the maximum setting. Further comparison with the newer designs of conventional colonoscope, which have more flexible shafts, will be of interest. Future studies should examine the usefulness of the VS colonoscope in training, in nonsedated colonoscopy, and in combination with new imaging techniques such as magnetic endoscope imaging.

1 Saunders BP, Macrae F, Williams CB. What makes colonos-

copy difficult? Gut 1993;34:A181.
2 Bat L, Williams CB. Usefulness of paediatric colonoscopes Bat L, Williams CB. Usefulness of paediatric colonoscopes
in adult colonoscopy. Gastrointest Endosc 1989;35:329-32.

3 Ness RM, Gottlieb K, Rex DK, Lehman GA. Difficult sigmoid colon intubation: guidewire exchange technique. Gastrointest Endosc 1996;44:99-101.

4 Rogers BHG. The use of small calibre endoscopes in selected cases increases the success rate of colonoscopy. Gastrointest Endosc 1989;35:352.

5 Williams CB. Split overtube for easier colonoscopy. Gastrointest Endosc 1983;24:A492.

6 Shinya H, Wolff WI. Colonoscopy. Surg Annu 1976;862: 257-95.

7 Kitano A, Okawa K, Obata A, et al. The leading cord method of colonofibreoscopy. Dis Colon Rectum 1986;29: 882-4.

8 Deyhle P, Demling L. Coloscopy-technique, results, indications. Endoscopy 1971;3:143-51.

9 Sullivan MJ. Variable stiffening device for colonoscopy. GasSullivan MJ. Variable stiffening devi
trointest Endosc 1990;36:642-3.

10 Ruffolo TA, Lehman GA, Rex D. Colonoscope damage from internal straightener use. Gastrointest Endosc 1991;37: 107-8.

11 Saunders BP, Fukumoto M, Halligan S, et al. Why is colonoscopy more difficult in women? Gastrointest Endosc 1996; 43:124-6.

12 Rex D, Imperiale TF, Portish V. Patients willing to try colonoscopy without sedation: associated clinical factors and results of a randomised controlled trial. Gastrointest Endosc 1999;49:554-9.

13 Hull T, Church JM. Colonoscopy-how difficult, how painful? Surg Endosc 1994;8:784-7

14 Shah SG, Saunders BP, Brooker JC, Williams CB. What happens during routine colonoscopy? An audit using mag-
netic positional imaging (MPI). Gastrointest Endosc 1999; 49:AB175.

15 Saunders BP, Bladen J, Williams CB, Bell GD. First clinical results with a real-time electronic imager as an aid to results with a real-time electronic
colonoscopy. Gut 1995;36:913-17.

16 Nelson DB, McQuaid KR, Bond JH, Macrae F. Populationbased colonoscopy screening for colorectal cancer is feasible and safe: preliminary results from the VA colonoscopy screening trial. Gastrointest Endosc 1999;49:AB65. 